Running Head: SELF-FOCUS IN NEUROTICISM

\title{
Interpersonal Cognitive Self-Focus as a Function of Neuroticism: Basal Tendencies and Priming Effects
}

\author{
Adam K. Fetterman and Michael D. Robinson \\ North Dakota State University
}

Note: Correspondence can be sent to Adam Fetterman, Psychology Department (Dept. 2765), PO Box 6050, North Dakota State University, Fargo, ND 58108-6050. Internet correspondence can be directed to Adam.Fetterman@ my.ndsu.edu (Phone: 701-231-9411; Fax: 701-231-8426). 


\begin{abstract}
Self-focus is one mechanism that may account for the social-evaluative anxiety of individuals high in neuroticism. The present two studies (total $N=183$ ) sought to cognitively model interpersonal self-focus. The cognitive task was a simple one in which participants simply categorized dyadic interpersonal pronouns, with reaction times as the dependent measure. When others engage us, the pronoun "me" refers to the other and the pronoun "you" refers to the self. Study 1 found a neuroticism by pronoun interaction on categorization time consistent with implicit interpersonal self-focus at high (but not low) levels of neuroticism establishing a basal tendency. Study 2 examined boundary conditions. Individuals high in neuroticism exhibited implicit self-focus particularly to the extent that they had been primed to think of themselves as submissive rather than dominant in their interpersonal interactions. Implications for understanding neuroticism, self-focus, and relationship functioning are discussed.
\end{abstract}

KEYWORDS: Neuroticism, Self-Focus, Interpersonal, Implicit, Anxiety 


\section{Interpersonal Cognitive Self-Focus as a Function of Neuroticism: \\ Basal Tendencies and Priming Effects}

Introduction

Neuroticism is predictive of numerous negative emotional states (Watson, 2000) and clinical conditions characterized by negative emotional states (Widiger, Verheul, \& van den Brink, 1999). Neuroticism is also predictive of substance abuse and suicide attempts (Lahey, 2009). Many of the outcomes predicted by neuroticism might be understood in terms of higher levels of self-focus, sometimes labeled self-consciousness or self-awareness. Self-focus is typically aversive and tends to magnify negative emotional states (Mor \& Winquist, 2002). Tendencies related to self-focus - such as rumination - predispose individuals toward clinical levels of anxiety and depression (Nolen-Hoeksema, 2000). Substance abuse may be precipitated by aversive self-focus (Arnett, 2005) and suicide attempts may represent the ultimate escape from aversive self-focus (Baumeister, 1990).

Consistent with such points, trait measures of self-focus often load onto a neuroticism factor (McCrae \& Costa, 1999). Further results are consistent with this mapping. Neuroticism is a positive predictor of trait worry (Muris, Roelofs, Rassin, Franken, \& Mayer, 2005), conceptualized in terms of repetitive self-focused processing concerning possible future events (Borkovec \& Sharpless, 2004). Neuroticism is a positive predictor of trait measures of rumination (Trapnell \& Campbell, 1999), conceptualized in terms of repetitive self-focused thinking about past events (Nolen-Hoeksema, 2000). Finally, there is some evidence for the idea that trait measures of worry and rumination mediate at least some of the pernicious consequences of neuroticism (Muris et al., 2005). 
Whether trait measures capture the dynamics of self-focus is arguable, however. As originally conceived, self-focus is a state, not a trait (Duval \& Wicklund, 1972). There is considerable evidence for this idea, in that several manipulations of self-focus have been shown to alter momentary levels of it (Carver \& Scheier, 1981). From an assessment-related perspective, it is doubtful that individuals can accurately report on whether they are self-focused or environment-focused at any point in time as such states are likely to be too fleeting and mercurial to be amenable to self-report methods (Dehaene, Changeux, Naccache, Sackur, \& Sergent, 2006; Posner \& Rothbart, 2007). For such reasons, we developed a novel cognitive assessment of self-focus, one that sought to model interpersonal dynamics.

When others communicate, they use the pronoun "me" to represent themselves and the pronoun "you" to reference the recipient of the communication - i.e., the self (Burgoon, Johnson, \& Koch, 1998). One only has to think about dyadic partners using the word "you" to appreciate this point. Following precedent (Fetterman \& Robinson, 2010; Fetterman, Robinson, \& Gilbertson, submitted), we therefore designed a simple cognitive task in which individuals were asked to quickly categorize such pronouns. The computer was essentially the interaction partner, consistent with a large body of work showing that people conceptualize computers in such terms (Nass \& Moon, 2000). This is of course advantageous in cognitively modeling self-focus. Momentary self-focus in this task would thus consist of faster categorizations of the pronoun "you" relative to "me" when presented on a computer screen.

\section{Hypotheses}

Study 1 sought to assess basal relations between neuroticism and cognitive self-focus. We hypothesized that neuroticism would interact with pronoun type to predict categorization speed. More specifically, we hypothesized that individuals high (but not low) in neuroticism 
would be faster to categorize the self-relevant interaction pronoun (YOU) relative to the otherrelevant interaction pronoun (ME). Findings of this type would support the idea that high levels of neuroticism covary with high levels of self-focus, but importantly so in an implicit cognitive manner.

Self-focus is quite malleable, however (Carver \& Scheier, 1981; Wicklund, 1979). Accordingly, Study 2 sought to examine the malleability of relations between neuroticism and cognitive self-focus. Toward this end, we randomly assigned individuals to write about a situation in which they were dominant versus submissive in the past prior to assessing cognitive self-focus. Submissive interactions tend to engender self-focus, among other effects (Lee-Chai \& Bargh, 2001). Accordingly, we hypothesized that individuals higher in neuroticism would exhibit higher levels of cognitive self-focus (and thus be faster to categorize the pronoun YOU relative to $\mathrm{ME}$ ) particularly to the extent that they were primed with submissive rather than dominant thoughts on the basis of autobiographical recall.

\section{Study 1}

The focus of Study 1 was on basal relations between neuroticism and cognitive selffocus. Accordingly, no priming manipulations occurred. We hypothesized that higher levels of neuroticism would be associated with greater levels of cognitive self-focus, defined in terms of relatively faster categorizations of the self-relevant pronoun relative to the other-relevant pronoun.

\section{Method}

\section{Participants and Assessment Procedures}

Participants were 103 (60 female) undergraduate volunteers from North Dakota State University who received course credit. They were run in groups of 6 or less on personal 
computers. The cognitive task was administered first to preclude trait-related priming effects on implicit task performance (Robinson \& Neighbors, 2006).

\section{Cognitive Self-Focus Task}

The task consisted of 120 trials and the program used Eprime software. On each trial, the word "YOU" or "ME" was presented at center screen on a randomized basis, with replacement. Participants were asked to categorize the pronoun as "you" or "me" using a serial response box as quickly and accurately as possible. Mappings were counterbalanced such that half of the participants were to press the 1 key in response to one pronoun and the other half of the participants were to press the 5 key in response to the same pronoun. Such mappings were displayed on the computer screen to reduce memory load. Stimuli varied in font size (either 15.5 or 20.5 Times New Roman font), but such variations were merely to keep the task "interesting" and are not relevant to the present predictions.

We sought to ensure a high degree of accuracy on the task so that categorization times would be the more informative measure (Sanders, 1998). Accordingly, we penalized inaccurate categorizations with a $1000 \mathrm{~ms}$ "Incorrect!” visual error message. By contrast, accurate categorizations were followed by a brief $150 \mathrm{~ms}$ delay prior to the next trial. Categorization accuracy was accordingly quite high in the task $(M=97 \%)$.

Categorization times were handled in a standard manner (Robinson, 2007). Trials associated with inaccurate responses were dropped. Categorization times were then logtransformed to reduce positive skew (Ratcliff, 1993). Finally, log-transformed times 2.5 SDs faster and slower than the mean were replaced by such $2.5 S D$ outlier values. Such transformed categorization times were then averaged as a function of the within-subject ME versus YOU design. Raw millisecond times were similarly averaged for presentation purposes. 


\section{Neuroticism Assessment}

Participants completed Goldberg's (1999) 10-item broad-bandwidth neuroticism scale, which correlates very highly with the NEO-PI neuroticism scale (Costa \& McCrae, 1992), other neuroticism scales as well (John \& Srivastava, 1999), and has exhibited excellent reliability and predictive validity in a number of studies (Goldberg et al., 2006). In more specific terms, participants were asked to rate the extent $(1=$ very inaccurate; $5=$ very accurate $)$ to which 8 statements characteristic of high levels of neuroticism (e.g., "get stressed out easily") and 2 statements characteristic of low levels of neuroticism (e.g., "am relaxed most of the time") generally describe the self. The latter two items were reverse-scored and a composite score was created by averaging across items (alpha $=.90 ; M=2.48 ; S D=0.79)$.

\section{Results}

Neuroticism was hypothesized to moderate the speed with which the pronouns were categorized in a manner suggesting greater cognitive self-focus at higher levels of neuroticism. This interactive hypothesis was examined in a General Linear Model (GLM) analysis as a function of the within-subject factor of pronoun (YOU versus ME) and z-scored variations in neuroticism (Aiken \& West, 1991), with log-transformed categorization time means as the dependent measure. There was no main effect for Neuroticism in this analysis, $F<1$, nor was there a main effect for Pronoun, $F<1$. Of more importance, the Neuroticism by Pronoun interaction was significant, $F(1,102)=4.03, p<.05$, partial eta squared $=.04$. Estimated millisecond means for the interaction at low (-1 SD) versus high $(+1 S D)$ levels of neuroticism (Aiken \& West, 1991) are reported in Figure 1.

Figure 1 indicates that high levels of neuroticism were predictive of cognitive self-focus (i.e., faster YOU than ME categorization times), whereas low levels of neuroticism were 
predictive of cognitive other-focus (i.e., faster ME than YOU categorization times). Follow-up analyses were performed. The within-subject effect was transformed into a difference score (Wilkowski \& Robinson, 2007): ME log latencies minus YOU log latencies. We then performed two simple regressions in which neuroticism levels were altered to reflect high $(+1 S D)$ versus low (-1 SD) levels of it. At high levels of neuroticism, the intercept was marginally significant and positive, $t(102)=1.68, p<.10$, such that this level of neuroticism was associated with marginally greater cognitive self-focus. At low levels of neuroticism the intercept was not significant, but was negative in sign, $t(102)=-1.17, p<.25$. What we particularly emphasize is the significance of the cross-over interaction, which best captures the dimensional trend toward greater levels of cognitive self-focus at higher levels of neuroticism.

\section{Discussion and Study 2}

The interactive results of Study 1 support a qualitatively different interpersonal focus from low (other-focused) to high (self-focused) levels of the trait of neuroticism. Such results confirm and extend neuroticism's purported link to self-focus, previously examined primarily on the basis of trait self-reports of self-consciousness and related constructs. Recall, though, that self-focus is almost certainly a malleable entity (Carver \& Scheier, 1981; Duval \& Silvia, 2002). In the present context, we suggest that individuals high in neuroticism are likely to exhibit cognitive self-focus particularly to the extent that they are uncertain or in self-doubt concerning their interpersonal standing. Consistent with this point, behavioral ecology (Mazur, 2005) and social cognition (Keltner, Gruenfeld, \& Anderson, 2003) literatures converge on the idea that interpersonal submissiveness is particularly likely to exacerbate self-focus among predisposed individuals. Accordingly, we randomly assigned Study 2 participants to write about either submissive or dominant interactions from the past. We hypothesized that this manipulation 
would interact with neuroticism to predict cognitive self-focus and that neuroticism would predict cognitive self-focus particularly in the submissive priming condition.

\section{Method}

\section{Participants and Assessment Procedures}

Participants were 80 (22 female) undergraduates from North Dakota State University who received course credit. Laboratory procedures were identical to Study 1. For example, participants were again run in groups of 6 or less and the cognitive task was completed first. The cognitive task was also identical to that previously described and categorization times were quantified exactly as in Study $1(M$ accuracy $=96 \%)$. Trait levels of neuroticism were again assessed in terms of the Goldberg (1999) scale (alpha $=.92 ; M=2.71 ; S D=0.97)$.

\section{Priming Manipulation}

Emotion states are frequently manipulated by short-duration (typically about 5 minute) tasks in which individuals recall and write about autobiographical memories of a given emotional type (Martin \& Clore, 2001). Such writing manipulations have also proven useful in manipulating states of power versus powerlessness, with predictable consequences for stereotyping and social cognition (Goodwin, Operario, \& Fiske, 1998; Guinote, 2008). Similar autobiographic recall plus writing procedures were used in the present study.

Individuals randomly assigned to the dominance condition were instructed to write about "a time in your life when you felt socially DOMINANT - that is, totally in power, able to do whatever you wanted to with confidence, capable of leading people to follow your wishes or plans..." Those assigned to the submission condition, on the other hand, were instructed to write about "a time in your life when you felt socially SUBMISSIVE - that is, totally powerless, incapable of doing what you wanted to do, dependent on another person's plans or wishes..." 
Writing prompts were as similar as possible across the two conditions and participants were to write for 5 minutes concerning details of the situation, their behaviors in the situation, and implications of the situation for their personal relationships. The written protocols were examined for content and it was clear that the events recalled were of the desired type and varied systematically by priming condition. After 5 minutes had elapsed, the E-Prime program informed participants that they were finished with the autobiographical memory task and would now be asked to complete a very different categorization task (i.e., that assessing cognitive self-focus).

\section{Results}

Log-transformed categorization times were analyzed in a GLM as a function of pronoun type (YOU versus ME), z-scored variations in neuroticism, and the between-subjects manipulation of priming condition. The main effect for Priming Condition was significant, $F$ (1, $78)=4.09, p<.05$, partial eta squared $=.05$ (a medium effect: Cohen, 1988), such that categorization times were faster in the submission priming condition $(M=424 \mathrm{~ms})$ than the dominance priming condition $(M=444 \mathrm{~ms})$. The effect might represent compensatory efforts toward activity following submissive states. The main effect for Neuroticism was not significant, $F<1$. Finally, there was a marginally significant main effect for Pronoun, $F(1,78)=3.43, p<$ .10 , partial eta squared $=.05$ (a medium effect: Cohen, 1988), such that the self-relevant pronoun (YOU) was categorized somewhat more quickly $(M=432 \mathrm{~ms})$ than the other-relevant pronoun (ME: $M=436 \mathrm{~ms}$ ). We attribute this marginal main effect to the general nature of the priming procedures, which induced a focus on the self by asking for autobiographical memories.

Two-way interactions involving condition were not significant, $F \mathrm{~s}<1$. The Neuroticism by Pronoun interaction was not significant either, $F(1,78)=2.27, p<.15$, partial eta squared $=$ .03, but it was in the same direction as Study 1 - i.e., cognitive self-focus was greater at higher 
levels of neuroticism. The non-significance of the interaction, though, supports the idea that the cognitive self-focus characteristic of high levels of neuroticism is malleable. Of most importance, then, was the significant Condition by Neuroticism by Pronoun interaction, $F(2,78)$ $=8.29, p<.01$, partial eta squared $=.10$ (a medium to large effect: Cohen, 1988).

We hypothesized that submissive interpersonal states, in particular, would activate selffocus at high levels of neuroticism. To examine this idea, we conducted follow-up GLM analyses, one particular to the dominance priming condition and one particular to the submission priming condition. Among those primed by dominant thoughts, there was no Neuroticism by Pronoun interaction, $F<1$ (at high neuroticism levels, estimated $M \mathrm{~s}=441 \& 440$ for the ME and YOU conditions; at low neuroticism levels, estimates $M s$ were $452 \& 444)$. By contrast, among those primed by submissive thoughts, the Neuroticism by Pronoun interaction was pronounced, $F(1,40)=10.33, p<.01$, partial eta squared $=.21($ a large effect: Cohen, 1988). Figure 2 displays estimated millisecond means for the submission priming condition at low $(-1 S D)$ versus high $(+1 S D)$ levels of neuroticism. The interaction was exactly of the cross-over type found in Study 1. It should be noted that due to the overall simple nature of the task, the differences in the means presented seem small but resulted in medium to large effect sizes (Cohen, 1988).

As in Study 1, but particular to the submission priming condition, we calculated a difference score to reflect the within-subject manipulation: ME log latencies minus YOU log latencies. We then performed two simple regressions in which neuroticism levels were altered to reflect high (+1 SD) versus low (-1 SD) levels of this trait (Wilkowski \& Robinson, 2007). At the high level of neuroticism, the intercept was significant, $t(42)=2.45, p<.05$, such that there was clear evidence for cognitive self-focus in the pronoun categorization task. At the low level of neuroticism, the intercept was marginally significant and - perhaps more importantly - reversed 
in direction, $t(42)=-1.79, p<.10$. The findings of Study 2 thus replicate those of Study 1 while further suggesting that submissive interpersonal states exacerbate cognitive self-focus at high, but not low, levels of neuroticism.

\section{Discussion}

Neuroticism is predictive of multiple forms of negative emotional reactivity (Gross, Sutton, \& Ketelaar, 1998; Suls \& Martin, 2005). Such forms of reactivity may be mediated, in part, by the more malleable self-views of high neuroticism individuals as neuroticism is predictive of self-uncertainty (Trapnell \& Campbell, 1999) and greater self-esteem instability across days (Kernis, 2005). The results of Study 2 are entirely novel, but they do reinforce the idea that the self is a more malleable entity at high, relative to low, levels of neuroticism. Submissiveness engendered cognitive self-focus only among individuals high in neuroticism, consistent with their greater reactivity to problematic events generally and self-focused reactions to such events in particular (Muris et al., 2005).

\section{General Discussion}

\section{Background and Findings}

Trait measures of neuroticism and self-consciousness systematically correlate with each other (e.g., McCrae \& Costa, 1999), though this is not universally true - e.g., in relation to trait measures of reflection rather than rumination (Trapnell \& Campbell, 1999). Of more importance, though, self-focus is a state rather than a trait according to seminal self-awareness literatures (Carver \& Scheier, 1981; Wicklund, 1979). Unfortunately, though, there are preciously few assessment-related probes of state self-focus and the probes that do exist are insufficiently cognitive and mostly of historical interest (e.g., Exner, 1973). 
Accordingly, we developed a novel cognitive probe of state self-focus, one embedding self-focus within an interactive, communication-related context (Burgoon et al., 1998). Study 1 examined basal relations between neuroticism and cognitive self-focus and found higher levels of it at higher levels of neuroticism. Study 2, though, found that neuroticism predicted cognitive self-focus particularly so following the activation of submissive thoughts. In the General Discussion section that follows, we focus on the nature of our task, implications of the findings, and present future directions of research.

\section{Task Considerations, Implications, and Directions for Future Research}

When people speak about themselves, they use the pronoun "me" to refer to the self and the pronoun "you" to refer to another person. The representational nature of these pronouns reverses, though, when communications come from another person (Burgoon et al., 1998). In such contexts, the pronoun "me" represents the communication partner and the pronoun "you" represents the target of the communication - i.e., the self. We sought to model such otheroriented communications using a computer because research has shown that computers are implicitly viewed as interaction partners (Nass \& Moon, 2000). In a variant of the present computerized task, furthermore, Fetterman et al. (submitted) confirmed that the pronoun "me" was seen to represent the computer and the pronoun "you" was seen to represent the self. Future studies following such communication context considerations are warranted. Even so, our results can only be interpreted in terms of the mappings confirmed by Fetterman et al. in that there is no basis for thinking that self-focus should be greater at low, rather than high, levels of neuroticism (McCrae \& Costa, 1999; Robinson \& Tamir, 2011; Silvia \& Duval, 2001).

Self-focus in interaction contexts may be generally problematic as it is likely to engender behaviors that favor the self's interests over the interaction partner's interests (Finkel \& Rusbult, 
2008). From this perspective, the present results have explanatory value. We found that high levels of neuroticism were differentially associated with cognitive self-focus, which in turn can help explain why the personal relationships of high neuroticism individuals are objectively more conflicted and more likely to lead to relationship dissolution, including divorce (Shackelford \& Buss, 1997; Smith, Traupman, Uchino, \& Berg, 2010). Further research, though, is necessary to establish this point more definitively.

More broadly, we suggest that our cognitive self-focus measure may have utility in future studies. Study 2 showed that performance in the task was malleable and therefore other manipulations thought to result in greater or lesser self-focus might be usefully modeled in the present cognitive terms. For example, rumination manipulations (e.g., Rusting \& NolenHoeksema, 1998) should result in greater cognitive self-focus. Cognitive self-focus should also be examined in relation to other individual differences. For example, psychopathy has been viewed in terms of a lack of appreciation for the needs and wishes of others (Zolondek, Lilienfeld, Patrick, \& Fowler, 2006) and should result in a similar pattern of cognitive self-focus. By contrast, especially agreeable or dependent individuals may exhibit other-focus in the task (Bornstein, 2005). Finally, there is potential interest in what type of personality would lead to increased self-focus in the dominance priming condition. It may be that personalities marked by self-aggrandizing (e.g., narcissism: Paulhus \& Williams, 2002) may lead to higher levels of a reflective self-focus in response to thinking about a time in which they were dominant. In fact, the task seems suited to understand object-relation dynamics of the sort long-theorized by psychodynamic clinicians (Baldwin, 1992), in general. 


\section{References}

Aiken, L., \& West, S. (1991). Multiple regression: Testing and interpreting interactions. Thousand Oaks, CA: Sage Publications, Inc.

Arnett, J. (2005). The developmental context of substance use in emerging adulthood. Journal of Drug Issues, 35, 235-254.

Baldwin, M. (1992). Relational schemas and the processing of social information. Psychological Bulletin, 112, 461-484.

Baumeister, R. F. (1990). Suicide as escape from self. Psychological Review, 97, 90-113.

Borkovec, T. D., \& Sharpless, B. (2004). Generalized anxiety disorder: Bringing cognitivebehavioral therapy into the valued present. In: S. C. Hayes, V. M. Follette, \& M. M. Linehan (eds.), Mindfulness and acceptance: Expanding the cognitive-behavioral tradition (pp. 209-242). New York: Guilford Press.

Bornstein, R. F. (2005). The dependent patient: A practitioner's guide. Washington, DC: American Psychological Association.

Burgoon, J.K., Johnson, M.L., \& Koch, P.T. (1998). The nature and measurement of interpersonal dominance. Communication Monographs, 65, 308-335.

Carver, C. S., \& Scheier, M. F. (1981). Attention and self-regulation: A control theory approach to human behavior. New York: Springer-Verlag.

Cohen, J. (1988). Statistical power analysis for the behavioral sciences (2nd ed.). Hillsdale, NJ: Lawrence Erlbaum Associates, Inc.

Costa, P. T., \& McCrae, R. R. (1992). Revised NEO Personality Inventory (NEO PI-R) and NEO Five-Factor Inventory (NEO-FFI). Professional manual. Lutz, FL: Psychological Assessment Resources. 
Dehaene, S., Changeux, J., Naccache, L., Sackur, J., \& Sergent, C. (2006). Conscious, preconscious, and subliminal processing: A testable taxonomy. Trends in Cognitive Sciences, 10, 204-211.

Duval, S., \& Wicklund, R. A. (1972). A theory of objective self awareness. Oxford England: Academic Press.

Duval, T., \& Silvia, P. J. (2002). Self-awareness, probability of improvement, and the selfserving bias. Journal of Personality and Social Psychology, 82, 49-61.

Exner, J. E. (1973). The self focus sentence completion: A study of egocentricity. Journal of Personality Assessment, 37, 437-455.

Fetterman, A. K. \& Robinson, M. D. (2010). Contingent self-importance among pathological narcissists: Evidence from an implicit task. Journal of Research in Personality, 44, 691697.

Fetterman, A. K., Robinson, M. D., \& Gilbertson, E. P. (submitted). Implicit self-importance in an interpersonal pronoun categorization task.

Finkel, E., \& Rusbult, C. (2008). Prorelationship motivation: An interdependence theory analysis of situations with conflicting interests. In: J. Y. Shah, \& W. L Gardner (eds.), Handbook of motivation science (pp. 547-560). New York: Guilford Press.

Goldberg, L. R. (1999). A broad-bandwidth, public domain, personality inventory measuring the lower-level facets of several five-factor models. In: I. Mervielde, I. Deary, F. De Fruyt, \& F. Ostendorf (eds.), Personality psychology in Europe (Vol. 7, pp. 7-28). Tilburg, The Netherlands: Tilburg University Press. 
Goldberg, L. R., Johnson, J. A., Eber, H. W., Hogan, R., Ashton, M. C., Cloninger, C. R., et al. (2006). The international personality item pool and the future of public-domain personality measures. Journal of Research in Personality, 40, 84-96.

Goodwin, S., Operario, D., \& Fiske, S. (1998). Situational power and interpersonal dominance facilitate bias and inequality. Journal of Social Issues, 54, 677-698.

Gross, J., Sutton, S., \& Ketelaar, T. (1998). Relations between affect and personality: Support for the affect-level and affective reactivity views. Personality and Social Psychology Bulletin, 24, 279-288.

Guinote, A. (2008). Power and affordances: When the situation has more power over powerful than powerless individuals. Journal of Personality and Social Psychology, 95, 237-252.

John, O. P., \& Srivastava, S. (1999). The Big Five trait taxonomy: History, measurement, and theoretical perspectives. In: L. A. Pervin, \& O. P. John (eds.), Handbook of personality: Theory and research (2nd ed., pp. 102-138). New York: Guilford Press.

Keltner, D., Gruenfeld, D.H., \& Anderson, C. (2003). Power, approach, and inhibition. Psychological Review, 110, 265-284.

Kernis, M. (2005). Measuring self-esteem in context: The importance of stability of self-esteem in psychological functioning. Journal of Personality, 73, 1569-1605.

Lahey, B. B. (2009). Public health significance of neuroticism. American Psychologist, 64, 241256.

Lee-Chai, A. \& Bargh, J. (Eds.). (2001). The use and abuse of power: Multiple perspectives on the causes of corruption. New York: Psychology Press.

Martin, L. L. \& Clore, G. L. (Eds). (2001). Theories of mood and cognition: A user's guidebook. Mahwah, N.J.: Lawrence Erlbaum Associates. 
Mazur, J. (2005). Biosociology of dominance and deference. Oxford England: Rowman \& Littlefield Publishers, Inc.

McCrae, R. R., \& Costa, P. R. (1999). The five-factor theory of personality. In O. P. John, R. W. Robins, L. A. Pervin, O. P. John, R. W. Robins, L. A. Pervin (eds.), Handbook of personality psychology: Theory and research (3rd ed.) (pp. 159-181). New York: Guilford Press.

Mor, N., \& Winquist, J. (2002). Self-focused attention and negative affect: A meta-analysis. Psychological Bulletin, 128, 638-662.

Muris, P., Roelofs, J., Rassin, E., Franken, I., \& Mayer, B. (2005). Mediating effects of rumination and worry on the links between neuroticism, anxiety and depression. Personality and Individual Differences, 39, 1105-1111.

Nass, C., \& Moon, Y. (2000). Machines and mindlessness: Social responses to computers. Journal of Social Issues, 56, 81-103.

Nolen-Hoeksema, S. (2000). The role of rumination in depressive disorders and mixed anxiety/depressive symptoms. Journal of Abnormal Psychology, 109, 504-511.

Paulhus, D., \& Williams, K. (2002). The Dark Triad of personality: Narcissism, Machiavellianism and psychopathy. Journal of Research in Personality, 36, 556-563.

Posner, M. I., \& Rothbart, M. K. (2007). Research on attention networks as a model for the integration of psychological science. Annual Review of Psychology, 58, 1-23.

Ratcliff, R. (1993). Methods for dealing with reaction time outliers. Psychological Bulletin, 114, $510-532$. 
Robinson, M. D. (2007). Lives lived in milliseconds: Using cognitive methods in personality research. In: R. W. Robins, R. C. Fraley, \& R. Krueger (eds.), Handbook of research methods in personality psychology (pp. 345-359). New York: Guilford Press.

Robinson, M. D., \& Neighbors, C. (2006). Catching the mind in action: Implicit methods in personality research and assessment. In: M. Eid \& E. Diener (eds.), Handbook of multimethod measurement in psychology (pp. 115-125). Washington, DC: American Psychology Association.

Robinson, M. D., \& Tamir, M. (2011). A task-focused mind is a happy and productive mind: A processing perspective. In: K. M. Sheldon, T. B. Kashdan, \& M. F. Steger (eds.), Designing positive psychology: Taking stock and moving forward (pp. 160-174). New York: Oxford University Press.

Rusting, C. L., \& Nolen-Hoeksema, S. (1998). Regulating responses to anger: Effects of rumination and distraction on angry mood. Journal of Personality and Social Psychology, 74, 790-803.

Sanders, A. F. (1998). Elements of human performance: Reaction processes and attention in human skill. Mahwah, NJ: Lawrence Erlbaum Associates Publishers.

Shackelford, T. K., \& Buss, D. M. (1997). Anticipation of marital dissolution as a consequence of spousal infidelity. Journal of Social and Personal Relationships, 14, 793-808.

Silvia, P. J., \& Duval, T. (2001). Objective self-awareness theory: Recent progress and enduring problems. Personality and Social Psychology Review, 5, 230-241.

Smith, T. W., Traupman, E. K., Uchino, B. N., \& Berg, C. A. (2010). Interpersonal circumplex descriptions of psychosocial risk factors for physical illness: Application to hostility, neuroticism, and marital adjustment. Journal of Personality, 78, 1011-1036. 
Suls, J., \& Martin, R. (2005). The daily life of the garden-variety neurotic: Reactivity, stressor exposure, mood spillover, and maladaptive coping. Journal of Personality, 73, 14851510.

Trapnell, P. D., \& Campbell, J. D. (1999). Private self-consciousness and the five-factor model of personality: Distinguishing rumination from reflection. Journal of Personality and Social Psychology, 76, 284-304.

Watson, D. (2000). Mood and temperament. New York: Guilford Press.

Wicklund, R. A. (1979). The influence of self-awareness on human behavior. American Scientist, 67, 187-193.

Widiger, T. A., Verheul, R., \& van den Brink, W. (1999). Personality and psychopathology. In: L. A. Pervin, \& O. P. John (eds.), Handbook of personality: Theory and research (2nd ed., pp. 102-138). New York: Guilford Press.

Wilkowski, B., \& Robinson, M. (2007). Keeping one's cool: Trait anger, hostile thoughts, and the recruitment of limited capacity control. Personality and Social Psychology Bulletin, $33,1201-1213$.

Zolondek, S., Lilienfeld, S. O., Patrick, C. J., \& Fowler, K. A. (2006). The interpersonal measure of psychopathy: Construct and incremental validity in male prisoners. Assessment, 13, 470-482. 
Figure 1

Cognitive Self-Focus by Neuroticism, Study 1

Figure 2

Cognitive Self-Focus by Neuroticism in the Submission Priming Condition, Study 2 


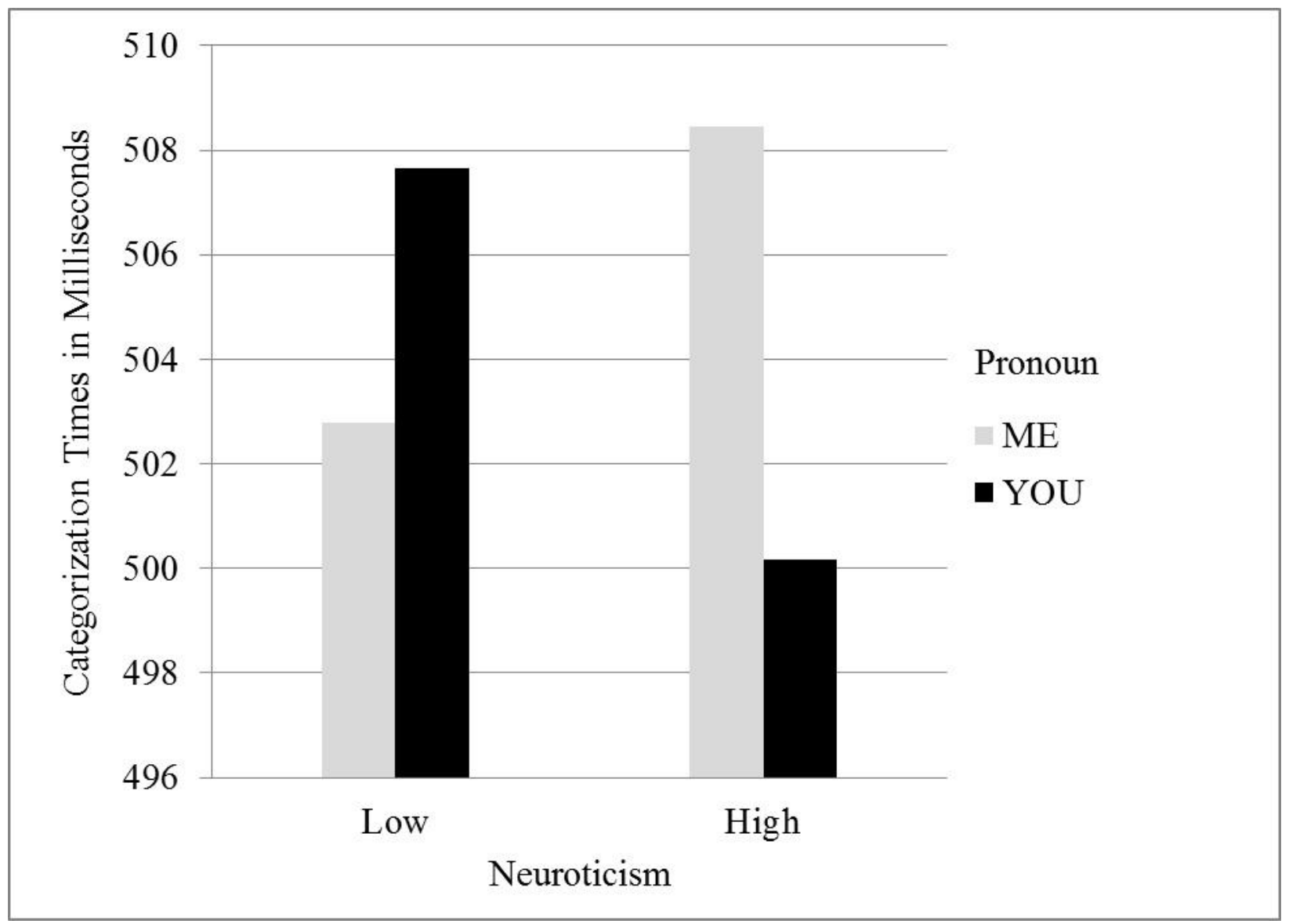




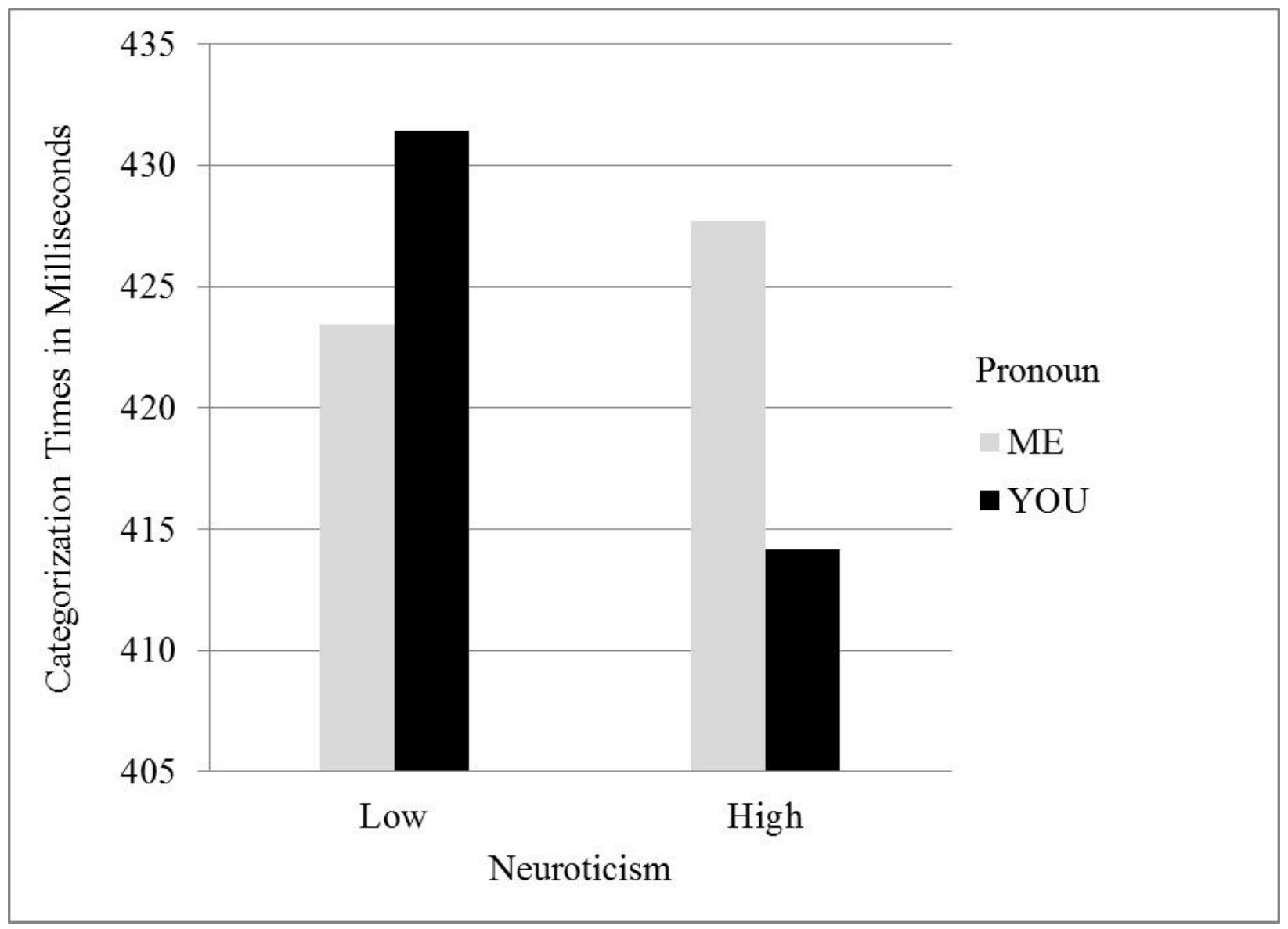

